\title{
Impact of Long-Term Antibiotic Use for Acne on Bacterial Ecology and Health Outcomes: A Review of Observational Studies
}

\author{
Jackie P-D. Garrett • David J. Margolis
}

Published online: 15 January 2012

(C) Springer Science+Business Media, LLC 2012

\begin{abstract}
Acne is among the most common cutaneous disorder affecting most individuals by the time they reach adulthood. Management of acne can be quite a challenge and includes medications with significant side effects. We discuss the potential side effects of long-term antibiotic use. These side effects should be carefully weighed and monitored when designing a treatment plan.
\end{abstract}

Keywords Acne - Antibiotic use - Epidemiology ·

Malignancy $\cdot$ Inflammatory bowel disease .

Collagen vascular illnesses

\section{Introduction}

Acne vulgaris is the most common cutaneous disorder affecting humans. This disorder most often affects the adolescent population. It typically resolves by adulthood; however, it may also start during adulthood. It is estimated that acne affects between $35 \%$ and $90 \%$ of adolescents. In adolescence, acne has a male predominance, although adult onset acne has a female predominance. Despite being a nonfatal disease, the

\section{J. P.-D. Garrett}

The Department of Pediatrics, Division of Allergy and Immunology,

The Children's Hospital of Philadelphia,

3550 Market Street,

Philadelphia, PA 19104, USA

e-mail: garrettjp@email.chop.edu

D. J. Margolis $(\square)$

Department of Biostatistics and Epidemiology and Dermatology,

The Perelman School of Medicine at the University of Pennsylvania, 815 Blockley Hall, 3400 Spruce Street,

Philadelphia, PA 19104, USA

e-mail: margo@mail.med.upenn.edu psychological ramifications of the condition lead to emotional distress [1].Optimizing the treatment for acne continues to be a challenge, especially given the potential side effects of the typical regimens [2]. This article focuses on the potential consequences of long-term antibiotic use.

\section{Antibiotics and Acne}

Propionibacterium acnes (P. acnes), the microbe that resides and can be abundant in the pilosebaceous unit, is associated with the severity of acne. P. acnes is an anaerobic organism that is part of the normal skin flora [3]. Obstruction of the follicular opening of the pilosebaceous unit provides an ideal environment for these bacteria to grow. Obstruction of the follicular opening is associated with the inflammatory component of acne through various suggested mechanisms. P. acnes can metabolize sebaceous triglycerides into fatty acids. P. acnes, along with sebum and keratin, generate proinflammatory mediators that recruit $\mathrm{T}$ lymphocytes, neutrophils, and foreign body giant cells [3]. It activates TLR2 (toll-like receptor 2) on monocytes and neutrophils, which activates the innate immunity pathway, generating proinflammatory cytokines including interleukin-12 (IL-12), interleukin-8 (IL-8), and tumor necrosis factor (TNF) [4, 5].

Antibiotics are used in acne for both the eradication of $P$. acnes and for their anti-inflammatory properties. The antibiotics used in the management of acne include both topical and systemic forms. The topical agents are generally clindamycin, erythromycin, dapsone, and sodium sulfacetamide. Topical clindamycin and erythromycin are often used in combination with other agents, such as benzoyl peroxide, a nonantibiotic agent used for its antimicrobial properties [6]. Oral options include macrolides, tetracyclines, trimethoprim- 
sulfamethoxazole and clindamycin. However, the most commonly used oral antimicrobials to treat acne are the tetracyclines, which are the main focus of this article.

\section{Tetracyclines}

Tetracyclines are antibiotics that function through the inhibition of protein synthesis and have anti-inflammatory properties. These agents share similar diverse antibacterial spectrum against pathogens from families as diverse as Staphylococcus, Rickettsia, Chlamydia, Listeria, and Neisseria. Drugs in this category include tetracycline, doxycycline, and minocycline. Doxycycline and tetracycline should be taken on an empty stomach because absorption is affected by food, dairy products, iron, and antacids. Minocycline is an exception, where its intake is not limited by the aforementioned agents. Tetracyclines should not be given to pregnant women or those under the age of 9 years. Tetracyclines carry risk of reduced bone growth and discoloration of the permanent teeth. Lastly, these agents can be photosensitizing. It has been claimed that the most photosensitizing agent is doxycycline and the least is minocycline (http:www. Uptodate.com/contents/ treatment-of-acne-vulgaris).

\section{Consequences of Long-Term Antibiotic Use}

In the past 15 years, research has further elucidated additional consequences of long-term antibiotic use. Various observational studies have examined certain complications that can be seen in long-term antibiotic use that may not be directly related to bacterial resistance. These studies describe increased infections, collagen vascular diseases, autoimmune diseases, and cancer. Observational studies by design cannot determine causation, only association. However, it is important to realize that many adverse events of interest are uncommon and occur several years after exposure, making it impossible to understand their natural history or association with an exposure using a randomized clinical trial design. In the remainder of this article, we highlight various studies that focus on each potential complication as a possible consequence of the use of antibiotics to treat acne.

\section{Antimicrobial Resistance}

Antimicrobial resistance is the result of excessive antibiotic use in the ambulatory setting [7]. Even with proper use of antibiotics to treat acne vulgaris, resistance can occur. Patel et al. [3] discussed the following factors that could influence the development of antibiotic resistance in patients with acne. These include antibiotic monotherapy, long-term administration of antibiotics, indiscriminate use outside their indications, inappropriate dosing, and the administration of antibiotics without concurrent use of benzoyl peroxide or topical retinoids [3].

\section{Infections}

Change in antimicrobial resistance patterns is the most frequent effect of long-term antibiotic use. In terms of infectious outcomes, patients on long-term antibiotics for acne seem to have a higher incidence of upper respiratory tract infections. In 2005, Margolis et al. [8] performed a retrospective cohort study using the United Kingdom General Practice Research Database. That database contains medical record information from general practitioners. The authors reviewed the data spanning from 1987 through 2002. Of the 84, 977 individuals, $71.7 \%$ required a topical or an oral antibiotic. During the first year of observation, $15.4 \%$ $(18,281)$ of those individuals with acne suffered from at least one upper respiratory tract infection. An association was found between patients with acne who were treated with antibiotics seeking medical care for upper respiratory tract infections as compared to those with acne who were not treated with antibiotics. Specifically, the odds of an upper respiratory tract infection developing in an individual with acne receiving antibiotics was 2.15 times greater than in one who was not receiving antibiotics $(P<0.001)$. Additional analyses compared acne patients with those who had other common medical illness, in an attempt to assure that the diagnosis of upper respiratory tract infections was not increased due to health care-seeking behavior. These analyses confirmed the result. The authors concluded that patients receiving antibiotic treatment were more likely to develop an upper respiratory tract infection [8]. Explanations for this finding include a change in the cutaneous flora distal from site of application and change in nasal colonization for those who are receiving both topical and oral antibiotics. In a subsequent article, the authors examined the likelihood that household contacts are at an increased risk for pharyngitis. This study also utilized the United Kingdom General Practice Research Database but focused on those that shared a residence with a patient diagnosed with acne. They found that exposure to an acne patient receiving antibiotics did not increase the contact's risk of developing an upper respiratory tract infection (odds ratio $0.94 ; 95 \% \mathrm{CI}, 0.89-1.0 ; P=0.063$ ) [9]. This association was again confirmed by a study that utilized both a cross-sectional design and a prospective cohort design. In these studies of college students, once again it was noted that those exposed to antibiotics for acne were more likely to report upper respiratory tract infections. In the crosssectional study, the odds ratio associating antibiotic use in acne patients with self-reported pharyngitis was 3.53 (95\% CI, 1.14-10.95). In the cohort study, the odds ratio associating antibiotic use and pharyngitis was 4.34 (95\% CI, 1.51-12.47) 
In both settings, the odds of reporting pharyngitis in those with acne on oral antibiotics were three times greater than those not receiving antibiotics. However, the study was not able to find an association between bacterial colonization with Group A streptococcus or Streptococcus salivarius and the administration of antibiotics used to treat acne nor the onset of pharyngitis $[10 \bullet \bullet$.

Although all of these studies consistently demonstrate an association between the use of antibiotics to treat acne and upper respiratory tract infections, they are limited because the diagnosis of pharyngitis is somewhat subjective, and it is possible that those with more severe acne (i.e. those more likely to receive antibiotics) are more likely to seek medical care for symptoms associated with the diagnosis of pharyngitis. Furthermore, the studies were not able to link a causative organism with the onset of pharyngitis. For example, two organisms that could potentially serve as causes of pharyngitis are Staphylococcus aureus and Streptococcus pyogenes. They are both sensitive to tetracyclines.

Staphylococcus aureus is a gram-positive, catalasepositive, and coagulase-positive bacterium. It is estimated that $40 \%$ to $50 \%$ of the general population is colonized. It can be isolated from the skin and the nasal cavity. S. aureus is somewhat fastidious, as it can survive on dry environmental surfaces for hours to days. It is also found on household pets, such as dogs, cats, and even horses. Methicillinresistant $S$. aureus (MRSA), however, only colonizes approximately $5 \%$ of the population $[11 \bullet \cdot]$. S. Aureus causes disease when the skin barrier is breached. It can cause local skin infections such as furuncles, carbuncles, or impetigo. More severe diseases include staphylococcal-scalded skin syndrome, bacteremia, osteomyelitis, and pneumonia. In the atopic dermatitis population, $S$. aureus super infection is more of a problem. The compromised skin barrier allows for infection with this organism. It is estimated that one half to one third of the patients with acne are colonized with $S$. aureus. Interestingly, those with acne being treated with tetracyclines do not appear to develop resistance [11]. In fact, those receiving tetracycline antibiotics to treat their acne were less likely to be colonized by $S$. aureus then those not receiving these oral antibiotics.

Streptococcus pyogenes, also known as Group A streptococcus (GAS), is another gram-positive coccus. It is a catalase-negative organism. GAS can cause mild infections like pharyngitis or impetigo and more invasive systemic illnesses such as necrotizing fasciitis or streptococcalscalded skin syndrome. Asymptomatic carriers tend to harbor the organism in the throat, nose, anus, and skin folds. Penicillin remains the drug of choice for treatment of GAS. Although GAS is associated with pharyngitis, it is not associated with pharyngitis in those with acne on antibiotics. In the original Margolis et al. [8] study examining incidence of upper respiratory tract infections in patients with acne treated with antibiotics, GAS was rarely found to colonize the upper respiratory tract of those with acne and was not found more frequently in those on tetracycline antibiotics.

\section{Collagen Vascular Illnesses}

Collagen vascular diseases have also been linked to the use of antibiotics for the treatment of acne. The most frequent case reports for adverse events with minocycline immediaterelease tablets are drug hypersensitivity, autoimmune effects, and hyperpigmentation [12]. Drug-induced lupus has been reported to be a complication of the use of tetracyclines. The pathogenesis of drug-induced lupus remains poorly understood. In a recent literature review on Schur et al. (http://www.uptodate.com/contents/drug-inducedlupus) discuss the possible pathogenetic causes for druginduced lupus. There were a few theories, such as 1) drug metabolites disrupt central immune tolerance; 2) cytotoxic drug metabolites cause pathology; 3 ) drugs act as haptens or agonists for drug-specific T cells; 4) abnormalities in oxidative drug metabolism; 5) abnormalities in thymus function; and 6) drugs nonspecifically activate lymphocytes. The end result is the induction of autoantibodies with the manifestation of autoimmunity in a particular population. With respect to those with acne, it is thought to be the result of two possible phenomena. The first is the exacerbation of underlying lupus symptoms. The second is the induction of lupus in a predisposed patient. The incidence of druginduced lupus is 15,000 to 30,000 cases per year [13]. It is equally common in males and females, with a predilection for older individuals and whites.

Interestingly the rate of disease differs by gender and by drug exposure. Drug-induced lupus among those with acne is more common among users of minocycline and in young females. Minocycline, unlike other tetracyclines, has been associated with the onset of drug-induced lupus. This association, as aforementioned, is most common in young women. The typical symptoms that patients report are rash, arthralgia, fever, and arthritis. However, myalgias and serositis can also occur. Autoantibodies associated with minocycline-induced lupus are ANA and P-ANCA. A particular complication that is seen in these patients is abnormal liver function. This has been detected in up to $50 \%$ of patients with minocycline-induced lupus [13]. Also, most patients' symptoms will resolve with drug discontinuation. Resolution usually occurs between 1 and 7 months after stopping therapy; $70 \%$ of the cases resolve within 2 to 3 months after discontinuation [14]. The exception is with young children, who possibly have an increased risk of prolonged disease.

Early case reports of minocycline-associated lupus first suggested this gender difference. In 1999, a nested casecontrol study by Sturkenboom et al. [15] looked more 
carefully at the association of tetracycline exposure and the development of lupus like syndrome. Their study was of 27,688 acne patients aged 15 to 29 years in the United Kingdom. They found that the incidence rates for males and females differed significantly: 2.3 cases per 100,000 (95\% CI, 0.07-13.0) prescriptions for males and 32.7 cases per 100,000 (95\% CI, 14.9-62.1) prescriptions for females (relative risk [RR], 14; 95\% CI, 1.8-111). Among the family of tetracyclines, the rateof lupus-like syndrome in females was 17.2 cases per $100,000(95 \%$ CI, 2.1-62.1) prescriptions for oxytetracycline, and 52.8 cases per $100,000(95 \%$ CI, 19.4-115) prescriptions for minocycline (RR, 3.1; 95\% CI, 0.6-15.2). One limitation of the study was that $93 \%$ of the patients were female [15].

In 2007, Margolis et al. [16] performed a retrospective cohort study of individuals aged 15 to 35 years in the UK whose general physicians subscribe to The Health Information Network (THIN) database. The study's goal was to look at the incidence of lupus in those with acne who were prescribed antibiotics in the tetracycline family. The study did support the previous data that shows minocycline, and no other tetracyclines, are associated with Lupus. They also found that the risk of developing lupus was greater after a total of 300 days of use or exposure to more than $50,000 \mathrm{mg}$ of the drug, and that it often required treatment. The overall hazard ratio for the association of minocycline to lupus was $2.64(95 \%$ CI, 1.51-4.66) and when adjusted for age and gender was 3.11 (95\% CI, 1.77-5.48). The limitation of this study includes its retrospective status, as the authors did not have the opportunity to examine the patients and agree to the diagnosis of lupus [16]. Another recently published article attempted to quantify the association between many drugs, including minocycline, and the development of lupus. This nested case-control study using the UK General Practice Research database looked at cases between 1997 and 2001. They also found a statistically increased risk of developing lupus with the use of minocycline (OR, 4.23; 95\% CI, 2.65-6.75). This was increased with increasing number of prescriptions as well (adjusted OR per 10 prescriptions was $3.10 ; 95 \% \mathrm{CI}, 2.10-4.55 ; P$ for trend $<0.001$ ) [17].

\section{Inflammatory Bowel Disease}

Gastrointestinal autoimmune diseases have also been linked to some acne treatments. Inflammatory bowel disease (IBD) has been seen with the use of isotretinoin (a vitamin A derivative). The incidence of IBD in this population is very small $(<1$ in 10,000$)$ [18]. One of the first observational studies to look at this association was by Bernstein et al. [19•]. This was a negative study, given that they were not able to show an association between isotretinoin use and IBD. Crocket et al. [20•], in a case-control study, showed an increased risk of ulcerative colitis, but not Crohn's disease, in those with isotretinoin exposure. Investigators at the University of Manitoba also performed a case-control study of patients with Crohn's disease or ulcerative colitis $[21 \bullet]$. Each case was matched with 10 controls. The study showed a lack of association between the use of Isotretinoin and the development of IBD. The incidence of IBD (1.2\%) in those who used isotretinoin was essentially equal to the incidence of IBD in those who did not use the drug (1.1\%).

In 2010, Margolis et al. [22] evaluated whether antibiotics used to treat acne could be associated with the development of IBD. Antibiotic use to treat acne could be an important confounder in that tetracycline use had previously been associated with IBD and it is more likely to be used to treat those with more severe acne (eg, such as those who might receive isotretinoin). Furthermore, patients prescribed isotretinoin generally have had some exposure to an extended course of antibiotics. The Margolis group performed a retrospective cohort study using the THIN database from the United Kingdom, which contains medical information of more than 5 million people. Their findings suggested an association with doxycycline use (adjusted hazard ratio $[\mathrm{HR}]=1.58 ; 95 \% \mathrm{CI}, 1.02-2.46$ ) and a possible association with tetracycline antibiotic use in the development of IBD in patients with acne (adjusted HR $=1.39 ; 95 \%$ CI, 1.02-1.90). This association was greatest for Crohn's disease and doxycycline exposure (specifically adjusted $\mathrm{HR}=2.25 ; 95 \% \mathrm{CI}$, 1.27-4.00). The other tetracyclines were moderately associated with Crohn's disease but not ulcerative colitis. Ultimately, the authors' recommended that one consider antibiotic exposure when assessing if treatment with other acne medications affects risk of developing inflammatory bowel disease [22]. It is important to note that the authors did perform analyses that controlled for oral contraceptive use a potential confounder for the female patients. The analyses showed no confounding effect.

Several studies have implicated oral contraceptives (OCP) in the development of ulcerative colitis and Crohn's disease. Cornish et al. [23] performed a random-effect metaanalysis comparing the incidence of ulcerative colitis or Crohn's disease between patients exposed and not exposed to oral contraceptives. The relative risk for ulcerative colitis in women currently taking the OCP was $1.53(95 \% \mathrm{CI}$, 1.21-1.94; $P=0.001)$ and 1.28 (95\% CI, 1.06-1.54; $P=0.011$ ), adjusted for smoking. The relative risk for Crohn's disease in women currently taking the OCP was $1.51(95 \% \mathrm{CI}, 1.17-1.96 ; P=0.002)$ and $1.46(95 \% \mathrm{CI}$, $1.26-1.70 ; P<0.001)$. The relative risk for Crohn's disease increased with the length of exposure to OCP, with a risk of 1.41 after 6 months of use and 3.48 after 3 years of use. The risk lost its significance upon discontinuation of the OCP [23]. 


\section{Malignancy}

Malignancies have been associated with the long-term use of antibiotics. The biological hypothesis for the association is that antibiotics alter intestinal microflora, which affects the synthesis of substances that protect against cancer. Also, antibiotics can alter the inflammatory milieu. Although limited, studies have focused on the association of antibiotic use with the risk of breast cancer. Velicer et al. [24] performed a case control study of women in the same health care plan. Their study found an increased risk of breast cancer among those who used antibiotics for any indication [24]. The study was not designed to fully elucidate why these women received the antibiotics and whether it was for an infection due to breast cancer [24]. In order to show that this risk was not due to infections caused by the malignancy, a subgroup analysis was performed of women who used macrolides and tetracyclines for acne and/or rosacea compared to those who were prescribed these medications for upper respiratory tract infections. This secondary analysis showed a similar increased risk of breast cancer in those with at least 50 days of exposure to antibiotics used to treat acne. Admittedly, the subgroup population examined was small. The authors suggestedthat more detailed analysis was needed, including timing of exposure and consideration of various antibiotic doses.

In 2006, Friedman et al. [25] performed a large retrospective chart review over a 9-year period also looking at the risk of breast cancer with chronic antibiotic use. They looked at individual drug groups, including tetracyclines, macrolides, and lincosamides. Again, an increased risk was noted for the tetracycline family (including minocycline, doxycycline, and tetracycline), and this association appeared to increase with dose. The risk was largest for those using antibiotics for greater than 101 days. The most common indication for the use of these drugs was acne or rosacea. The same result, however, occurred with exposure to macrolides (erythromycin, azithromycin and clarithromycin). The most common indication for these antibiotics was to treat upper respiratory tract infections. The lincosamides, namely clindamycin, did not show elevated risk with increased exposure. In essence, these drugs show a weak association and we cannot ascertain causality. The authors recommended additional longer follow-up studies to evaluate this association [25].

Studies have also looked at the risk of lung cancer after antibiotic exposure. Zhang et al. [26] conducted a nested casecontrol study in a population-based cohort using the THIN database in the United Kingdom. This study collected antibiotic information prospectively. In order to omit those patients who may display susceptibility to infection in the subclinical phase of their cancer, they used a lag time of 1 year. For all antibiotics, the greater the exposure (as measured by the number of prescriptions) the greater the increased risk of lung cancer, albeit this association was decreased after adjustment for confounders. When they further restricted the smoking status of subjects, the association decreased further. There was, however, no significant increased risk seen in users of erythromycin, trimethoprim or oxytetracycline. Their ultimate conclusion was that the increased risk found with use of antibiotics may be biased and in reality associated with other risk factors for lung cancer that might increase the likelihood of receiving an antibiotic prescription [26].

\section{Conclusions}

Acne is a widely prevalent disease that can be difficult to manage. Making the best decision for the appropriate treatment course may include the prolonged use of antibiotics. Albeit uncommon, it is important for the health care provider and patient to realize that long-term use of antibiotics to treat acne has been associated with important adverse events. Several of these adverse events were discussed in this review and include upper respiratory tract infection, which is likely a marker for alterations in the microbial milieu, collagen vascular illnesses, inflammatory bowel disease, and cancer. These adverse events are generally rare but have been demonstrated in more than one study. In any event, it is difficult to fully understand how these potential concerns should influence patient management. It is hoped that many of these issues will be better resolved with future study.

Disclosure No conflicts of interest relevant to this article were reported.

\section{References}

Papers of particular interest, published recently, have been highlighted as:

- Of importance

•• Of major importance

1. Bowe WP, Leyden JJ, Crerand CE, Sarwer DB, Margolis DJ. Body dysmorphic disorder symptoms among patients with acne vulgaris. J Am Acad Dermatol. 2007;57(2):222-30. Epub 2007/05/15.

2. Williams HC, Dellavalle RP, Garner S (2011) Acne vulgaris. Lancet. Epub 2011/09/02.

3. Patel M, Bowe WP, Heughebaert C, Shalita AR. The development of antimicrobial resistance due to the antibiotic treatment of acne vulgaris: a review. JDD. 2010;9(6):655-64. Epub 2010/07/22.

4. Kim J, Ochoa MT, Krutzik SR, Takeuchi O, Uematsu S, Legaspi AJ, et al. Activation of toll-like receptor 2 in acne triggers inflammatory cytokine responses. J Immunol. 2002;169(3):1535-41. Epub 2002/07/23. 
5. Webster GF. Inflammatory acne represents hypersensitivity to Propionibacterium acnes. Dermatology. 1998;196(1):80-1. Epub 1998/04/29.

6. Dutil M. Benzoyl peroxide: enhancing antibiotic efficacy in acne management. Skin Therapy Letter. 2010;15(10):5-7. Epub 2010/ $11 / 16$.

7. Gonzales R, Malone DC, Maselli JH, Sande MA. Excessive antibiotic use for acute respiratory infections in the United States. Clinical infectious diseases: an official publication of the Infectious Diseases Society of America. 2001;33(6):757-62. Epub 2001/08/21.

8. Margolis DJ, Bowe WP, Hoffstad O, Berlin JA. Antibiotic treatment of acne may be associated with upper respiratory tract infections. Arch Dermatol. 2005;141(9):1132-6.

9. Bowe WP, Hoffstad O, Margolis DJ. Upper respiratory tract infection in household contacts of acne patients. Dermatology. 2007;215(3):213-8.

10. • Margolis DJ, Fannelli M, Kupperman E, Papadopoulos M, Joshua $\mathrm{M}$ et al. Association of pharyngitis with oral antibiotic use for the treatment of acne: A cross-sectional and prospective cohort study. Archives of dermatology. Epub 2011/10/24. This is a study of the incidence of pharyngitis and antibiotic use for the treatment of acne. No association was noted with group A streptococcus.

11. •• Fanelli M, Kupperman E, Lautenbach E, Edelstein PH, Margolis DJ. Antibiotics, acne, and Staphylococcus Aureus colonization. Arch. Dermatol. 2011;147(8):917-21. Epub 2011/04/13. This is a study of the frequency of Staphylococcus aureus colonization among patients with acne. It examined the antibiotic susceptibility patterns between the patients who are using antibiotics and those who are not using antibiotics.

12. Leyden JJ, Del Rosso JQ. Oral antibiotic therapy for acne vulgaris: pharmacokinetic and pharmacodynamic perspectives. JCAD. 2011;4(2):40-7. Epub 2011/03/10.

13. Borchers AT, Keen CL, Gershwin ME. Drug-induced lupus. Ann New York Acad Sci. 2007;1108:166-82. Epub 2007/09/26.

14. Schlienger RG, Bircher AJ, Meier CR. Minocycline-induced lupus. A systematic review. Dermatology. 2000;200(3):223-31. Epub 2000/06/01.

15. Sturkenboom MC, Meier CR, Jick H, Stricker BH. Minocycline and lupuslike syndrome in acne patients. Arch Intern Med. 1999;159(5):493-7. Epub 1999/03/13.

16. Margolis DJ, Hoffstad O, Bilker W. Association or lack of association between Tetracycline class antibiotics used for acne vulgaris and lupus erythematosus. Br J Dermatol. 2007;157(3):540-6. Epub 2007/06/29.
17. Schoonen WM, Thomas SL, Somers EC, Smeeth L, Kim J, Evans $\mathrm{S}$, et al. Do selected drugs increase the risk of lupus? A matched case-control study. Br J Clin Pharmacol. 2010;70(4):588-96. Epub 2010/09/16.

18. Shale M, Kaplan GG, Panaccione R, Ghosh S. Isotretinoin and intestinal inflammation: what gastroenterologists need to know. Gut. 2009;58(6):737-41. Epub 2009/05/13.

19. • Bernstein CN, Nugent Z, Longobardi T, Blanchard JF. Isotretinoin is not associated with inflammatory bowel disease: a population-based case-control study. The American journal of gastroenterology. 2009;104(11):2774-8. Epub 2009/07/23. An association between isotretinoin use and inflammatory bowel disease was not noted in this study.

20. • Crockett SD, Porter CQ, Martin CF, Sandler RS, Kappelman MD. Isotretinoin use and the risk of inflammatory bowel disease: a casecontrol study. The American journal of gastroenterology. 2010;105 (9):1986-93. Epub 2010/04/01. An association between inflammatory bowel disease and isotretinoin use was noted in this study.

21. - Card T, Logan RFA, Rodrigues LC e t al. Antibiotic use and the development of Crohn's disease. Gut 2004;53:246-50. This is one of the first studies to describe the association between antibiotics and inflammatory bowel disease.

22. Margolis DJ, Fannelli M, Hoffstad O, Lewis J. Potential association between oral tetracycline class of antimicrobials used to treat acne and inflammatory bowel disease. Am J Gastroenterol. 2010;105(12):2610-6. Epub2010/8/10.

23. Cornish JA, Tan E, Simillis C, Clark SK, Teare J, et al. The risk of oral contraceptives in the etiology of inflammatory bowel disease: a meta-analysis. Am J Gastroenterol. 2008;103:2394-400.

24. Velicer CM, Heckbert SR, Lampe JW, Potter JD, Robertson CA, Taplin SH. Antibiotic use in relation to the risk of breast cancer. JAMA. 2004;291(7):827-35. Epub 2004/02/19.

25. Friedman GD, Oestreicher N, Chan J, Quesenberry CP, Jr., Udaltsova N, Habel LA. Antibiotics and risk of breast cancer: up to 9 years of follow-up of 2.1 million women. Cancer epidemiology, biomarkers \& prevention: a publication of the American Association for Cancer Research, cosponsored by the American Society of Preventive Oncology. 2006;15 (11):2102-6. Epub 2006/11/23.

26. Zhang H, Garcia Rodriguez LA, Hernandez-Diaz S. Antibiotic use and the risk of lung cancer. Cancer epidemiology, biomarkers \& prevention: a publication of the American Association for Cancer Research, cosponsored by the American Society of Preventive Oncology. 2008;17(6):1308-15. Epub 2008/06/12. 